\title{
Neuroprotective effect of therapeutic hypothermia versus standard care alone after convulsive status epilepticus: protocol of the multicentre randomised controlled trial HYBERNATUS
}

\author{
Stephane Legriel ${ }^{1,2^{*}}$, Fernando Pico ${ }^{3}$, Yves-Roger Tran-Dinh $^{4}$, Virginie Lemiale ${ }^{5}$, Jean-Pierre Bedos ${ }^{1}$, \\ Matthieu Resche-Rigon ${ }^{6,7,8}$ and Alain Cariou ${ }^{2,9,10}$
}

\begin{abstract}
Convulsive status epilepticus (CSE) is a major medical emergency associated with a $50 \%$ morbidity rate. CSE guidelines have recommended prompt management for many years, but there is no evidence to date that they have significantly improved practices or outcomes. Developing neuroprotective strategies for use after CSE holds promise for diminishing morbidity and mortality rates. Hypothermia has been shown to afford neuroprotection in various health conditions. We therefore designed a trial to determine whether 90-day outcomes in mechanically ventilated patients with CSE requiring management in the intensive care unit (ICU) are improved by early therapeutic hypothermia $\left(32-34^{\circ} \mathrm{C}\right)$ for $24 \mathrm{~h}$ with propofol sedation. We are conducting a multicentre, open-label, parallel-group, randomised, controlled trial (HYBERNATUS) of potential neuroprotective effects of therapeutic hypothermia and routine propofol sedation started within $8 \mathrm{~h}$ after CSE onset in ICU patients requiring mechanical ventilation. Included patients are allocated to receive therapeutic hypothermia $\left(32-34^{\circ} \mathrm{C}\right)$ plus standard care or standard care alone. We plan to enrol 270 patients in $11 \mathrm{ICUs}$. An interim analysis is scheduled after the inclusion of 135 patients. The main study objective is to evaluate the effectiveness of therapeutic hypothermia $\left(32-34^{\circ} \mathrm{C}\right)$ for $24 \mathrm{~h}$ in diminishing 90 -day morbidity and mortality (defined as a Glasgow Outcome Scale score $<5$ ). The HYBERNATUS trial is expected to a decreased proportion of patients with a Glasgow Outcome Scale score lower than 5 after CSE requiring ICU admission and mechanical ventilation.
\end{abstract}

Trial registration Clinicaltrials.gov identifier NCT01359332 (registered on 23 May 2011)

Keywords: Therapeutic hypothermia, Randomised controlled trial, Status epilepticus, Outcome, Intensive care unit

\section{Background}

Convulsive status epilepticus (CSE) is a life-threatening emergency that requires on-scene antiepileptic treatment adjusted according to the treatment response [1]. Hospital mortality is about $20 \%$ overall and reaches $40 \%$ in patients with severe refractory CSE [2]. The morbidity

\footnotetext{
*Correspondence: slegriel@ch-versailles.fr

${ }^{1}$ Medical-Surgical Intensive Care Unit, Centre Hospitalier de Versailles - Site André Mignot, 177 rue de Versailles, 78150 Le Chesnay Cedex, France Full list of author information is available at the end of the article
}

associated with CSE is more difficult to evaluate, as adverse events are often erroneously attributed to the cause of CSE. In retrospective studies, Glasgow Outcome Scale (GOS) scores indicated neurological impairments in $28 \%$ of patients after CSE and $54 \%$ after refractory CSE [3]. In a prospective multicentre study, $50 \%$ of CSE survivors requiring ICU admission had impaired 90-day functional outcomes defined as a GOS score $<5$ [4]. By multivariate analysis, factors independently associated with poorer outcomes were age, duration of motor 
seizures, focal neurological signs, and progression to refractory status epilepticus (SE) [4]. Some of these factors may be amenable to improvement and therefore may constitute therapeutic targets. Thus, outcomes may be improved by the rapid control of seizure activity (shorter seizure duration and prevention of refractory CSE and NCSE) and by treatment of the cause of CSE $[5,6]$.

Therapeutic hypothermia has shown both anticonvulsant and neuroprotective properties after $\mathrm{SE}$ in experimental studies. In rats, hypothermia at $32-34{ }^{\circ} \mathrm{C}$ decreased the incidence of both seizures and associated local and hippocampal neuronal damage [7-13]. In humans, therapeutic hypothermia has been described merely as an adjuvant treatment of super-refractory SE in adults and paediatric patients [14-17]. Finally, therapeutic hypothermia may also target some of the causes of status epilepticus such as ischaemic or haemorrhagic stroke [18-22] and subarachnoid haemorrhage [23-25]. Its use in patients with traumatic brain injury demonstrated conflicting results [26-30]. Thus, pathophysiological considerations and experimental data suggest a beneficial effect of therapeutic hypothermia in SE. Proof of such an effect in human patients has not been obtained [31, 32].

The main goal of the HYBERNATUS trial is to determine whether therapeutic hypothermia $\left(32-34{ }^{\circ} \mathrm{C}\right)$ for $24 \mathrm{~h}$ has neuroprotective effects compared to standard care alone. HYBERNATUS is a multicentre, open-label, parallel-group, randomised, controlled trial in patients with CSE requiring admission to the intensive care unit (ICU) and mechanical ventilation.

\section{Methods/design}

\section{Ethical aspects}

The study protocol was approved by the local independent ethics committee (Comité de Protection des Personnes CPP Ile de France IV, Saint Louis, on 1 September 2010, \#2010/27) and the competent French health authorities (AFSSAPS, on 22 June 2010, \#EudraCT 2010-A00466-33). Informed consent is obtained from each patient or next of kin prior to study inclusion. The trial was registered at Clinicaltrials.gov identifier NCT01359332 (registered on 23 May 2011).

\section{Aims}

The main study objective is to evaluate the effectiveness of therapeutic hypothermia $\left(32-34{ }^{\circ} \mathrm{C}\right)$ for $24 \mathrm{~h}$ in decreasing 90-day morbidity and mortality, defined as a GOS score $<5$, in patients with CSE requiring ICU admission and mechanical ventilation.

The secondary objectives are to evaluate the effectiveness of therapeutic hypothermia $\left(32-34{ }^{\circ} \mathrm{C}\right)$ for $24 \mathrm{~h}$ in decreasing seizure duration, progression to refractory CSE, ICU mortality, hospital mortality, 90-day mortality, hospital stay length, and 90-day functional impairments (motor and/or cognitive impairment, epilepsy).

\section{Design}

HYBERNATUS is a multicentre, open-label, parallelgroup, randomised, controlled trial in patients with CSE requiring ICU admission and mechanical ventilation. Patients are allocated at random to either early therapeutic hypothermia plus standard care or standard care alone at the acute phase of ICU management.

\section{Setting}

Among the 11 French ICUs participating in the study, 6 are in university hospitals. All participating ICU staff members have received training in the study procedures and in protocols for optimal CSE management.

\section{Study population}

Inclusion criteria are age over 18 years, admission to one of 11 participating ICUs, CSE (5 min or more of continuous clinical seizure activity or more than two seizures without a return to baseline in the interval), time since seizure onset no longer than $8 \mathrm{~h}$, and need for mechanical ventilation.

Exclusion criteria are a full recovery, need for emergent surgery (neurosurgery or other) precluding therapeutic hypothermia, postanoxic status epilepticus, dying patient, decision to withdraw or withhold life-sustaining treatments, and pregnancy.

All patients admitted to one of the participating ICUs with any seizure activity are screened for eligibility by the ICU physicians 24/7.

\section{Randomisation}

Consecutive eligible patients are randomly allocated in a 1:1 ratio to one of the two treatment groups. The randomisation scheme involves permuted blocks and stratification on centre, age ( $\leq 65$ or $>65$ years), and seizure duration ( $\leq 60 \mathrm{~min}$ or $>60 \mathrm{~min}$ ). Randomisation and concealment are ensured by using a secure, computer-generated, interactive, response system available at each study centre and managed by the biometrics unit of the Saint Louis University Hospital (Paris, France), which has no role in patient recruitment.

\section{Study interventions}

The study protocol and randomisation arms are detailed in Figs. 1 and 2 and Table 1.

\section{Standardised anticonvulsant treatments after randomisation in both groups}

To be eligible, patients should have received at least one of the first-line anticonvulsant drugs (clonazepam and 


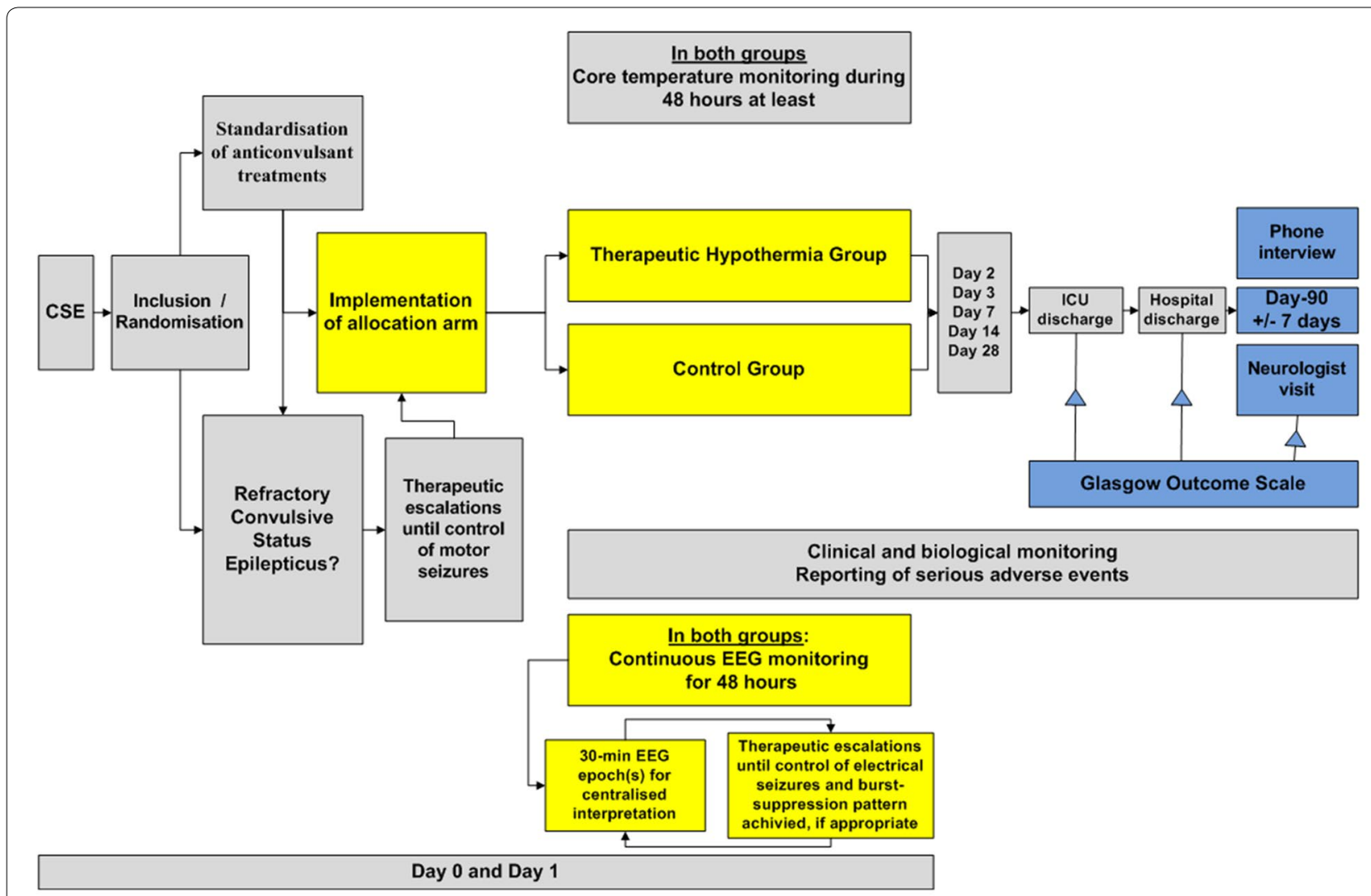

Fig. 1 HYBERNATUS trial diagram. ICU intensive care unit. EEG electroencephalogram

diazepam) listed in French guidelines. Patients with ongoing motor seizures who have not received a secondline anticonvulsant (fosphenytoin, phenytoin, phenobarbital, or sodium valproate) before randomisation receive a loading dose of one of these drugs selected according to the indications and specific contraindications of each [33].

Patients with refractory CSE, defined as CSE persisting despite two lines of anticonvulsant therapy within $60 \mathrm{~min}$, are given an intravenous $2 \mathrm{mg} / \mathrm{kg}$ propofol bolus followed by an additional intravenous $1 \mathrm{mg} / \mathrm{kg}$ bolus every 5 min until motor seizures stop, at which point a continuous intravenous propofol infusion is started at a rate of $2-5 \mathrm{mg} / \mathrm{kg} / \mathrm{h}$. In patients with uncontrolled refractory CSE despite continuous intravenous propofol at a rate of $5 \mathrm{mg} / \mathrm{kg} / \mathrm{h}$, anaesthetic drugs (midazolam or thiopental) can be added.

CSE must be controlled within 60 min after randomisation in both groups and before the induction of therapeutic hypothermia in the experimental group.

\section{Therapeutic hypothermia group}

The objective is to lower the core body temperature to $32-34{ }^{\circ} \mathrm{C}$ rapidly after randomisation and then to maintain this temperature for $24 \mathrm{~h}$. The management in the therapeutic hypothermia group will include the following:

- sedation with propofol in a 1-2 $\mathrm{mg} / \mathrm{kg}$ bolus followed by a maintenance infusion at a rate of $2-5 \mathrm{mg} / \mathrm{kg} / \mathrm{h}$; patients with refractory CSE before randomisation receive the propofol infusion rate that was required to stop the motor seizures;

- neuromuscular blockade with a $0.15 \mathrm{mg} / \mathrm{kg}$ bolus of cisatracurium or of another neuromuscular blocker followed by doses at 4-h intervals adjusted to maintain the response to train-of-four stimulation at two of four twitches;

- hypothermia induction with ice-cold intravenous fluids at $4{ }^{\circ} \mathrm{C}$ and then hypothermia maintenance with ice packs at the groin and neck and a cold-air tunnel around the patient's body;

- after $24 \mathrm{~h}$ of hypothermia, passive rewarming by covering the patient with a warm blanket, at a slow rate of $0.25-0.5{ }^{\circ} \mathrm{C}$ per hour to avoid haemodynamic instability, cisatracurium is discontinued when normothermia is achieved; when the train-of-four response is four twitches, the propofol is stopped or 


\section{Verification of inclusion criteria}

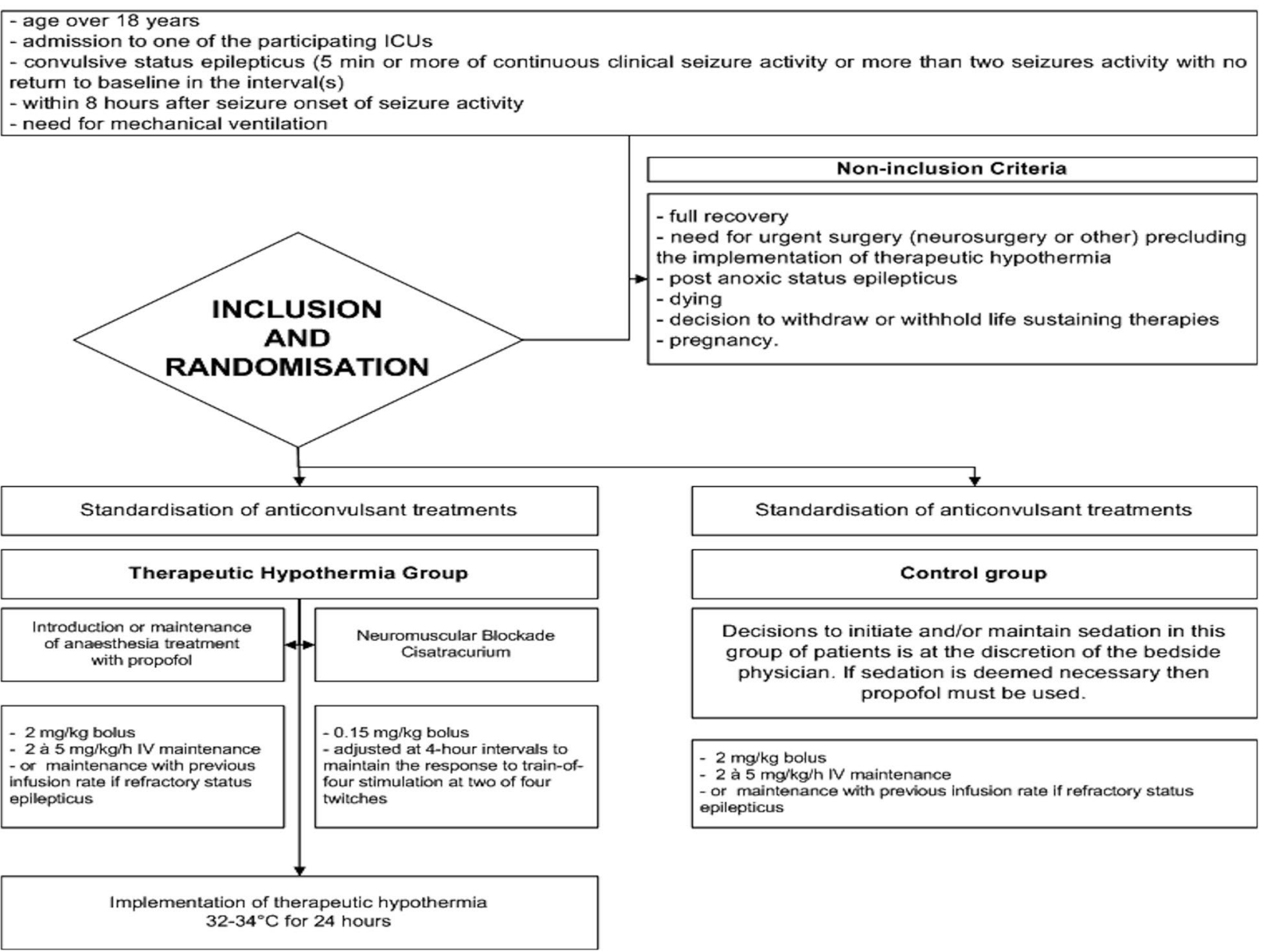

Fig. 2 Eligibility criteria, inclusion, randomisation, and treatment implementation modalities in each arm of the trial

decreased to the lowest dose needed to ensure tolerance of the endotracheal tube;

- If continued sedation is required, the choice of the sedative is at the discretion of the bedside physician, but propofol is not used for longer than $48 \mathrm{~h}$;

- Core body temperature is monitored using an oesophageal probe throughout the therapeutic hypothermia phase and then during rewarming until two values $4 \mathrm{~h}$ apart are between $37^{\circ}$ and $38^{\circ} \mathrm{C}$.

\section{Control group}

The bedside physician decides whether sedation should be initiated and maintained. If sedation is given, the same propofol regimen as in the therapeutic hypothermia group is used (1-2 mg/ $\mathrm{kg}$ bolus and then $2-5 \mathrm{mg} /$ $\mathrm{kg} / \mathrm{h}$ intravenous infusion rate). If sedation is inadequate with continuous intravenous propofol $5 \mathrm{mg} /$ $\mathrm{kg} / \mathrm{h}$, benzodiazepines may be added. Finally, if sedation must be maintained beyond $48 \mathrm{~h}$, the propofol must be stopped and an anaesthetic selected by the bedside physician given instead.

\section{General management of convulsive status epilepticus (CSE) in both groups}

In both control and intervention groups, a continuous EEG monitoring is started within $2 \mathrm{~h}$ after randomisation and maintained during the first $48 \mathrm{~h}$ after randomisation and/or until body temperature normalisation in the therapeutic hypothermia group. A 30-min standard EEG extracted from the continuous EEG recording is sent for centralised interpretation by a qualified neurophysiologist within $2 \mathrm{~h}$ after the initiation of continuous EEG monitoring. If the results show refractory CSE or progression to NCSE, the patient is given a $2-\mathrm{mg} / \mathrm{kg}$ 
Table 1 HYBERNATUS trial flow chart

\begin{tabular}{|c|c|c|c|c|c|c|c|}
\hline & Inclusion & Day 0 & Day 1 & $\begin{array}{l}\text { Day } 2 \\
\text { Day } 7 \\
\text { Day } 14 \\
\text { Day } 28\end{array}$ & $\begin{array}{l}\text { ICU } \\
\text { discharge }\end{array}$ & $\begin{array}{l}\text { Hospital } \\
\text { discharge }\end{array}$ & $\begin{array}{l}\text { Day } 90 \\
\text { ( } \pm 7 \text { days) }\end{array}$ \\
\hline Eligibility: check inclusion and non-inclusion criteria & $x$ & & & & & & \\
\hline Patient/next of kin information and consent & $x$ & & & & & & \\
\hline Randomisation (within $8 \mathrm{~h}$ after CSE onset) & & $x$ & & & & & \\
\hline Standardised anticonvulsant treatment & & $x$ & & & & & \\
\hline Brain imaging and other aetiological investigations & & $x$ & & & & & \\
\hline Therapeutic hypothermia (in the intervention group) & & $x$ & $x$ & & & & \\
\hline Core temperature monitoring (every $4 \mathrm{~h}$ ) & & $x$ & $x$ & & & & \\
\hline Continuous EEG monitoring & & $x$ & $x$ & & & & \\
\hline 30-min continuous EEG extract (centralised reading) & & $x$ & $x$ & & & & \\
\hline Clinical and laboratory monitoring & & $x$ & $x$ & $x$ & $x$ & & \\
\hline Serious adverse event reporting & & $x$ & $x$ & $x$ & $x$ & $x$ & $x$ \\
\hline Glasgow Outcome Scale (GOS) score evaluation & & & & & $x$ & $x$ & $X^{1,2}$ \\
\hline Standard EEG & & & & & & & $x^{2}$ \\
\hline Brain MRI & & & & & & & $x^{2}$ \\
\hline MMSE score & & & & & & & $x^{2}$ \\
\hline FIM scale score & & & & & & & $x^{2}$ \\
\hline Neurological evaluation & & & & & & & $x^{2}$ \\
\hline
\end{tabular}

${ }^{1}$ By phone interview in all cases

${ }^{2}$ Neurologist visit, if feasible

propofol bolus, repeated every $5 \mathrm{~min}$ if necessary, and then a maintenance intravenous infusion at $2-5 \mathrm{mg} / \mathrm{kg} / \mathrm{h}$ to obtain and maintain a burst-suppression EEG pattern for $24 \mathrm{~h}$. Further EEG epochs are sent for centralised interpretation by a qualified neurophysiologist until a burst-suppression EEG pattern is observed.

Second-line anticonvulsant drugs administered intravenously are switched to enteral administration when feasible. Patients with a known history of epilepsy are given their previous treatment enterally or parenterally. Aetiological investigations are carried out early, simultaneously with the symptomatic and anticonvulsant treatment. They include a thorough initial physical examination and tests for metabolic disturbances (e.g. hypoglycaemia, hypocalcaemia, hyponatraemia, uraemia, hypomagnesaemia, hypoxaemia, and hypercapnia) and carbon monoxide poisoning. Computed tomography $(\mathrm{CT})$ of the brain is performed routinely. In patients with previously treated epilepsy, serum anticonvulsant drug assays are performed if appropriate, to determine whether levels are within the therapeutic range. As dictated by the clinical setting, tests are performed for disorders such as porphyria and thyroid dysfunction and/or for toxic substances (alcohol, cocaine, amphetamines, tricyclic, or serotonergic antidepressants). A lumbar puncture is performed in patients with any of the following: fever, meningeal neck stiffness, immunodeficiency, and negative findings from the other aetiological investigations. Magnetic resonance imaging (MRI) may be considered if all other aetiological investigations are negative [34].

\section{Other general measures}

In both groups, a standardised neurological examination is performed every $4 \mathrm{~h}$ by the physicians and nurses in charge of the patient (GOS score, pupillary reflex, corneal reflex, cough reflex, spontaneous breathing, abnormal movements, focal neurological signs). Core body temperature is monitored continuously using an oesophageal probe and recorded every $4 \mathrm{~h}$ during the first $48 \mathrm{~h}$ after randomisation.

Patients are routinely screened for propofol infusion syndrome via measurements of arterial blood gas, serum lactate, serum triglycerides, and serum creatine phosphokinase every $8 \mathrm{~h}$ during the first $48 \mathrm{~h}$ after randomisation.

The prevention of secondary brain injury relies on temperature control to avoid hyperthermia in the control group and, after rewarming, in the therapeutic hypothermia group; glucose control between 0.8 and $1.4 \mathrm{~g} / \mathrm{L}$, maintenance of normoxia with $\mathrm{PaO}_{2} \geq 80 \mathrm{mmHg}$ and $\mathrm{SaO}_{2} \geq 95$ \%; maintenance of normocapnia with $\mathrm{PCO}_{2}$ between 35 and $40 \mathrm{mmHg} ; 45^{\circ}$ head-of-bed elevation; maintenance of mean arterial pressure between 70 and $90 \mathrm{mmHg}$; and serum sodium control between 
138 and $142 \mathrm{mmol} / \mathrm{L}$. Special attention is paid to the occurrence of aspiration pneumonia, and three samples for blood cultures are taken routinely within $24 \mathrm{~h}$ after randomisation.

\section{Safety and potential risks associated with the study}

Rigorous monitoring procedures are implemented to minimise the risks associated with therapeutic hypothermia in patients after CSE. Continuous EEG monitoring is performed for $48 \mathrm{~h}$ after inclusion, and a 30-min EEG extract is interpreted centrally before implementation of the randomly allocated treatments. Although neuromuscular blockade can theoretically mask recurrent seizure activity, this risk is lessened by the anticonvulsant properties of therapeutic hypothermia.

Preliminary data show that $82 \%$ of patients admitted to the ICU for CSE receive mechanical ventilation for longer than $24 \mathrm{~h}$, and that nearly $25 \%$ of those given mechanical ventilation for less than $24 \mathrm{~h}$ have a 90 -day GOS score lower than 5 . Thus, the use of mechanical ventilation for an unnecessarily long period seems very rare. No criteria are available for predicting which patients will require mechanical ventilation for less than $24 \mathrm{~h}$. Finally, this study includes only patients with severe CSE requiring both ICU admission and mechanical ventilation.

Patients receiving therapeutic hypothermia and/or propofol are closely monitored for adverse effects of these treatments such as infections and propofol infusion syndrome. We therefore believe that the use of therapeutic hypothermia does not expose the patients to undue risks, given the high morbidity and mortality rate in our prospective study [4] and the safety measures included in the HYBERNATUS trial protocol.

\section{Data collection and follow-up}

The following baseline characteristics are recorded at admission: age, sex, date and time of ICU admission, McCabe score and Knaus score, and pre-existing comorbidities. The Simplified Acute Physiology Score II is computed $24 \mathrm{~h}$ after ICU admission.

The study protocol also requires collection of the following data:

- Description of the CSE: circumstances of onset, date and time of onset and of seizure control, clinical features of seizures, pre-hospital and hospital providers and timing of anticonvulsant and supportive treatments, results of aetiological investigations (brain $\mathrm{CT}$, lumbar puncture, MRI, laboratory tests including toxicological screen), aetiological diagnosis, type and dosage of antiepileptic drugs, and date and start and stop times of continuous EEG monitoring;
- Data related to ICU and hospital management: description of the acute disease; description of organ failures according to the Logistic Organ Dysfunction score (at inclusion and on days $0,1,2,7$, 14 , and 28); use of mechanical ventilation, inotropic support, and/or renal replacement therapy; vital and functional status at ICU and hospital discharge according to the GOS; and cause of death (treatment limitation decision, refractory SE, and/or ICU complications);

- Data related to therapeutic hypothermia: modalities of induction and maintenance; core temperature every $4 \mathrm{~h}$ during the first $48 \mathrm{~h}$; serum levels of creatine phosphokinase, triglycerides, and electrolytes; arterial lactate and blood gas levels; electrocardiogram; occurrence of aspiration pneumonia; and results of blood cultures on samples taken within the first $24 \mathrm{~h}$;

- Data related to outcomes: 90-day GOS score by structured phone interview and neurologist visit; description of functional sequelae; mini-mental state examination (MMSE) score; and Functional Independence Measure scale (FIM) score.

\section{Organisation of the trial \\ Funding/support}

HYBERNATUS is fully sponsored by the Direction de la Recherche Clinique et du Développement (DRCD), Assistance Publique-Hopitaux de Paris, France, with a grant from the French Ministry of Health (Programme Hospitalier de Recherche Clinique, PHRC AOM 09 180). There is no industry sponsorship or funding.

\section{Coordination and conduct of the trial}

Before recruitment of the first patient, all physicians and other healthcare workers in the 11 participating ICUs attended formal training sessions on the study protocol, data collection in the case-report form (CRF), and implementation of continuous EEG monitoring. All documents required for the study, including the study protocol and management guidelines, are available in each participating ICU. In each participating ICU, the physicians and a clinical research nurse and/or clinical research assistant are in charge of daily patient screening and inclusion, ensuring compliance with the study protocol, and collecting the study data in the CRF. Finally, all participating physicians are available for inclusion and continuous EEG monitoring around the clock and 7 days per week. The DRCD clinical research unit regularly reviews all clinical data collected in the CRF. Any serious adverse event must be reported within $48 \mathrm{~h}$ after its occurrence. 


\section{Interim analyses}

An interim analysis is scheduled after the enrolment of 135 patients. The O'Brien-Fleming method will be used.

\section{Blinding}

Blinding of the physicians, nurses, and patients to the use of therapeutic hypothermia is not feasible. To minimise evaluation bias, outcomes will be evaluated during a structured phone interview by independent assessors blinded to the allocation group.

\section{Study outcomes}

\section{Primary outcome}

The primary outcome is GOS score determined by a structured interview lower than 5 on day 90 .

\section{Secondary outcomes}

Secondary outcomes are as follows:

- Percentages of patients with recurrent convulsive and/or non-convulsive seizures between 6 and $12 \mathrm{~h}$ after randomisation

- Total seizure duration in minutes

- Percentage of patients with refractory status epilepticus

- Mortality in the ICU, in the hospital, and by day 90

- Total ICU and hospital stay lengths in days

- Percentages of patients with functional impairments on day 90: frequency of seizures, recurrence of status epilepticus after discharge, number of antiepileptic drugs, MMSE score, and FIM score

\section{Modalities of outcome evaluation}

The GOS score is determined on day 90 ( \pm 7 days) during a structured phone interview conducted by an independent assessor blinded to the allocation group (Table 2).

When possible, on day 90 ( \pm 7 days), patients return to the centre where they were included, for an evaluation by a neurologist including an interview and determination of the GOS score by structured interview, MMSE score, and FIM score. The patient brings an MRI of the brain and conventional EEG. The neurologist records any recurrent CSE episodes since randomisation; frequency of any recurrent seizures, as well as their type and associated symptoms; nature and dosage of antiepileptic drugs; and other treatments.

\section{Independent data safety monitoring board (DSMB)}

The independent data safety monitoring board (DSMB) is composed of three physicians (Thomas De Broucker, Neurology Department, Saint Denis Hospital, Saint Denis, France; Alain Combes, Intensive Care Unit, Pitié Salpétrière University Hospital, France; and Nicolas
Pichon, Intensive Care Unit, Limoges University Hospital, Limoges, France) and one biostatistician (Patricia Jabre, Urgences-Samu 93, Avicenne University Hospital, Bobigny, France) not otherwise involved in the trial. The DSMB assesses the safety data whenever complete 90-day follow-up data become available for 45 additional patients. For each of these safety analyses, the DSMB has access to blinded results on 28-day mortality, ICU discharge mortality, and 90-day GOS scores. The DSMB also evaluates all reported serious adverse events.

\section{End of the trial}

Each patient is in the trial for 90 days. Premature study withdrawal is considered if requested by the patient or next of kin. Patients who are lost to follow-up or do not receive the randomly assigned treatment are not classified as prematurely withdrawn from the trial. Prematurely withdrawn patients are not replaced but undergo the procedures scheduled for the last visit, to the extent possible. Furthermore, the reason for premature withdrawal is recorded in both the CRF and the source document.

\section{Sample size}

The working hypothesis for this randomised controlled trial is that, compared to standard care alone, standard care combined with therapeutic hypothermia increases the proportion of patients with a good 90-day outcome defined as a GOS score of 5 . Assuming that $40 \%$ of patients will have a GOS score of 5 on day 90 in the standard care group [4] and that this percentage will increase by $20 \%$ (to $60 \%$ ) with therapeutic hypothermia, with a $5 \%$ two-sided type I error rate, $90 \%$ power, and a single interim analysis performed according to the O'Brien-Fleming method, 135 patients are needed in each group (270 patients in all).

\section{Statistical methods}

The statistical analysis will follow the intention-to-treat approach, with each patient being analysed as a member of the group assigned by randomisation, regardless of subsequent events. In addition, any missing value will be replaced by the previous value according to the last value carried forward method. A sensitivity analysis of missing values will be carried out, using multiple imputation by chained equations (MICE). Comparisons of the two groups will routinely consider a possible centre effect.

A statistical analysis report will be written to describe all the findings according to CONSORT statement recommendations, taking into account the specific features of the trial. Any change in the analysis plan will be justified in the final report. 
Table 2 Categories of the Glasgow Outcome Scale Adapted from Jennett B et al.

\begin{tabular}{|c|c|c|}
\hline Category & Classification & Description \\
\hline 1 & Death & Patient is certified dead \\
\hline 2 & Vegetative state & $\begin{array}{l}\text { Patient is unable to interact with the environment } \\
\text { Patients who show no evidence of meaningful responsiveness. This non-sentient state must be distinguished from other } \\
\text { conditions of wakeful, reduced responsiveness-such as the locked-in syndrome, akinetic mutism, and total global apha- } \\
\text { sia. Vegetative patients breathe spontaneously, have periods of spontaneous eye-opening, may follow moving objects } \\
\text { with their eyes, show reflex responses in their limbs (to postural or painful stimuli), and they may swallow food placed in } \\
\text { their mouths }\end{array}$ \\
\hline 3 & Severe disability & $\begin{array}{l}\text { Patient is unable to live independently but can follow commands } \\
\text { This indicates that a patient is conscious but needs the assistance of another person for some activities of daily living every } \\
\text { day. This may range from continuous total dependency to the need for assistance with only one activity }\end{array}$ \\
\hline 4 & Moderate disability & $\begin{array}{l}\text { Patient is capable of living independently but unable to return to work or school } \\
\text { Such a patient is able to look after himself at home, to get out, and about to the shops and to travel by public transport. } \\
\text { However, some previous activities, either at work or in social life, are now no longer possible by reason of either physical or } \\
\text { mental deficit }\end{array}$ \\
\hline 5 & Mild or no disability & $\begin{array}{l}\text { Patient is able to return to work or school } \\
\text { This indicates the capacity to resume normal occupational and social activities, although there may be minor physical or } \\
\text { mental deficits. However, for various reasons, the patient may not have resumed all his previous activities and in particu- } \\
\text { lar may not be working }\end{array}$ \\
\hline
\end{tabular}

\section{Primary outcome}

The primary outcome will be assessed by using logistic regression to compare the percentage of patients with a 90-day GOS score lower than 5 in the two study groups. If needed, the logistic regression model will be adjusted to allow a more accurate assessment of the effect.

\section{Secondary outcomes}

The method described above for the primary outcome will also be used for the percentages of patients with EEG evidence of an ongoing epileptic seizure between 6 and 12 h or of refractory SE after randomisation; ICU, hospital, and 90-day mortality; and 90-day functional impairments.

To compare seizure duration (in minutes), we will build a semi-parametric regression model (Cox or Fine-Gray) appropriate for an analysis of competing risks (control of SE vs. death without control of SE). This approach will be used also for ICU and hospital stay lengths (alive at ICU or hospital discharge vs. death in the ICU or hospital, respectively).

\section{Discussion}

SE is among the most common and challenging lifethreatening events in patients with neurological failure. Despite national and international guidelines emphasising the importance of urgent anticonvulsant therapy according to a stepwise algorithm, SE remains associated with high mortality and morbidity rates. The contrast between the dual anticonvulsant and neuroprotective properties of therapeutic hypothermia demonstrated in experimental studies, on the one hand, and the paucity of clinical evidence, on the other, combined with the potential beneficial effects of therapeutic hypothermia on several causes of SE, prompted us to design the HYBERNATUS trial [35].

Several key points of the trial design received special attention and deserve discussion. First, our focus on patients with CSE requiring ICU management is warranted by reports of severe functional impairments after CSE. Indeed, the main objective of the study is neuroprotection, as opposed to only seizure control. We hypothesised that early implementation of our neuroprotective strategy in comatose patients after the control of CSE would translate into improved 90-day outcomes. We therefore decided to include not only patients with refractory CSE, but also all patients remaining comatose after control of motor seizures. Similarly, we planned to include patients regardless of the underlying aetiology even if this may lead to heterogeneity. As we were unwilling to require the use of mechanical ventilation for the trial, we included only patients who needed mechanical ventilation for any medical reason after CSE. Second, the choice of $32-34{ }^{\circ} \mathrm{C}$ as the therapeutic hypothermia target rests on both data from experimental animal models of SE and current practice regarding the use of therapeutic hypothermia in various conditions such as coma after cardiac arrest, stroke, or even traumatic brain injury [27, $36,37]$. Finally, the 24-h duration of therapeutic hypothermia after CSE control is based on the current standard of care for refractory SE, which includes anaesthetics for up to $24 \mathrm{~h}$ with a burst-suppression EEG pattern [5, 32]. Third, therapeutic hypothermia requires sedation and neuromuscular blockade. To ensure patient awakening as early as possible after the end of the neuroprotective procedure, we chose drugs with short half-lives, 
such as propofol and cisatracurium. Propofol has the additional advantage of exerting anticonvulsant effects. However, we felt it would be unethical to require the use of sedation in patients in the control arm. Thus, the continuation of sedation in controls is at the discretion of the bedside physician. Finally, safety considerations led us to limit the use of propofol to $48 \mathrm{~h}$ with an infusion rate no greater than $5 \mathrm{mg} / \mathrm{kg} / \mathrm{h}$, in both arms [38]. Fourth, we chose the 90-day GOS score as the primary outcome. The GOS is used chiefly to evaluate overall outcomes of neurological conditions [39-41]. The GOS in its structured form has been found valid, practical, and reliable [42]. It also has the advantage of having been used in several studies of SE, and it is well suited to an assessment of the physical and functional burden after CSE [43]. Moreover, in the preliminary work that allowed us to formulate the hypothesis for the sample size estimation, the 90-day functional outcome was evaluated using the GOS [4]. Importantly, the GOS has been found valid, practical, and reliable $[42,44]$.

To conclude, one of the main strengths of the HYBERNATUS trial is the uniformity of the study population and careful standardisation of CSE management in both groups. All patients are managed according to the recent literature, with continuous EEG monitoring and a comprehensive aetiological workup. The only difference between the two groups is the use of therapeutic hypothermia at $32-34{ }^{\circ} \mathrm{C}$ for $24 \mathrm{~h}$, which is determined by randomisation. If the study hypothesis is confirmed, therapeutic hypothermia at $32-34{ }^{\circ} \mathrm{C}$ after control of CSE in patients requiring mechanical ventilation and ICU management will become part of standard practice in order to improve patient outcomes.

\section{Study protocol amendments and safety monitoring}

Following first communication of a study indicating that moderate hypothermia may be harmful in patients with severe bacterial meningitis [45], we submitted a protocol amendment to the local independent ethics committee (Comite de Protection des Personnes CPP Ile de France IV, Saint Louis, on 15 January 2013) to not enrol patients with bacterial meningitis. This change was implemented on 24 January 2013.

Based on the results of planned the interim analysis (May 2013), the DSMB recommended the continuation of patients recruitment.

\section{Trial status}

The trial has been validated and accepted for financial support by the French National Health Ministry (Programme Hospitalier de Recherche Clinique, PHRC AOM 09-P081249). Patient inclusion started in March 2011, and enrolments were completed on
January 2015. Data management and planned analysis are ongoing, and release of the results is planned for the end of 2016.

\begin{abstract}
Abbreviations
CRF: case-report form; CSE: convulsive status epilepticus; EEG: electroencephalography; GOS: Glasgow Outcome Scale; ICU: intensive care unit; NCSE: non-convulsive status epilepticus; SE: status epilepticus.
\end{abstract}

\section{Authors' contributions}

$S L$ and AC drafted the manuscript, and MRR prepared the statistical analysis and helped to draft the manuscript. All authors read and approved the final manuscript.

\begin{abstract}
Author details
${ }^{1}$ Medical-Surgical Intensive Care Unit, Centre Hospitalier de Versailles - Site André Mignot, 177 rue de Versailles, 78150 Le Chesnay Cedex, France.

${ }^{2}$ INSERM U970 (Team 4), Paris Cardiovascular Research Center, Paris, France. ${ }^{3}$ Neurology and Stroke Department, Centre Hospitalier de Versailles - Site André Mignot, 177 rue de Versailles, 78150 Le Chesnay Cedex, France. ${ }^{4}$ Neurophysiology Department, Hôpital Lariboisière, AP-HP, 2 rue Ambroise Paré, 75010 Paris, France. ${ }^{5}$ Medical Intensive Care Unit, Saint Louis University Hospital, AP-HP, Paris, France. ${ }^{6}$ SBIM Biostatistics and Medical information, Hôpital Saint-Louis, APHP, 1 avenue Claude Vellefaux, Paris, France. ${ }^{7}$ Université Paris Diderot, Paris, France. ${ }^{8}$ ECSTRA Team (Epidémiologie Clinique et Statistiques pour la Recherche en Santé), UMR 1153 INSERM, Université Paris Diderot, Sorbonne Paris Cité, Paris, France. ${ }^{9}$ Medical Intensive Care Unit, Cochin University Hospital, Hopitaux Universitaires-Paris Centre, AP-HP, Paris, France. ${ }^{10}$ Paris Descartes University, Sorbonne Paris Cité-Medical School, Paris, France.
\end{abstract}

\section{Acknowledgements}

This study is sponsored by the Direction de la Recherche Clinique et du Développement (DRCD), Assistance Publique-Hopitaux de Paris, France, and is supported by a grant from the French Ministry of Health (Programme Hospitalier de Recherche Clinique, PHRC AOM 09 180). The authors thank A. Wolfe for helping to prepare the manuscript. Neither the study sponsor nor the study funder had any role in designing the trial; managing, analysing, or interpreting the data; writing the report; or deciding to submit the report for publication; neither the study sponsor nor the study funder has authority over any of these activities.

Contributors-the HYBERNATUS study group Fabrice DAVIAUD (Cochin University Hospital), Nicolas MONGARDON (Cochin University Hospital), Virginie LEMIALE (Saint Louis University Hospital), Elie AZOULAY (Saint Louis University Hospital), Emmanuel CANET (Saint Louis University Hospital), Virginie LAURENT (Versailles Hospital), Gwenaelle JACQ (Versailles Hospital), Sybille MERCERON (Versailles Hospital), Jerome BEDEL (Versailles Hospital), Benjamin PLANQUETTE (Versailles Hospital), Julia Hilly-Ginoux (Versailles Hospital), Antoine GROS (Versailles Hospital), Nathalie ZAPPELLA (Versailles Hospital), Alexis FERRE (Versailles Hospital), Christelle SIMON (Versailles Hospital), Pierre GUEZENNEC (Versailles Hospital), Fabrice BRUNEEL (Versailles Hospital), Benjamin ZUBER (Versailles Hospital), Guillaume LACAVE (Versailles Hospital), Gilles TROCHE (Versailles Hospital), Jean-Denis MOYER (Versailles Hospital), David GRIMALDI (Versailles Hospital), Isabelle MALISSIN (Lariboisiere University Hospital), Nicolas DEYE (Lariboisiere University Hospital), Sophie RIGAUDEAU (Versailles Hospital), Benoit CREPON (Versailles Hospital), Herve MENTEC (Argenteuil Hospital), Marina THIRION (Argenteuil Hospital), Aicha HAMDI (Montreuil Hospital), Mohamed SRAIRI (Toulouse University Hospital), Thomas GEERAERTS (Toulouse University Hospital), David SCHNELL (Strasbourg University Hospital), Raluca NEAGU (Strasbourg University Hospital), Ferhat MEZIANI (Strasbourg University Hospital), Maleka SCHENCK (Strasbourg University Hospital), Jean-Etienne HERBRECHT (Strasbourg University Hospital), Jean-Yves LEFRANT (Nimes University Hospital), Benjamin LOUART (Nimes University Hospital), Jonathan CHELLY (Melun Hospital), Christophe VINSONNEAU (Melun Hospital), Thomas ROSSIGNOL (Le Mans Hospital), Sebastien CAVELOT (Versailles Hospital), Pernelle VAUBOIN (Saint Louis University Hospital), Lakhdar MAMERI (Saint Louis University Hospital), Nathalie MARIN (Cochin University Hospital). All authors have consented to publication of their information. 


\section{Competing interests}

The authors declare that they have no competing interests.

Received: 13 May 2016 Accepted: 6 June 2016

Published online: 21 June 2016

\section{References}

1. Lowenstein DHAB. Status epilepticus. N Engl J Med. 1998;1998(338):970-6.

2. Legriel S, Mourvillier B, Bele N, Amaro J, Fouet P, Manet P, et al. Outcomes in 140 critically ill patients with status epilepticus. Intensive Care Med. 2008;34:476-80

3. Claassen J, Lokin JK, Fitzsimmons BF, Mendelsohn FA, Mayer SA. Predictors of functional disability and mortality after status epilepticus. Neurology. 2002;8(58):139-42.

4. Legriel S, Azoulay E, Resche-Rigon M, Lemiale V, Mourvillier B, Kouatchet A, et al. Functional outcome after convulsive status epilepticus. Crit Care Med. 2010:38:2295-303.

5. Brophy GM, Bell R, Claassen J, Alldredge B, Bleck TP, Glauser T, et al. Guidelines for the evaluation and management of status epilepticus. Neurocrit Care. 2012;17:3-23.

6. Rossetti AO, Hurwitz S, Logroscino G, Bromfield EB. Prognosis of status epilepticus: role of aetiology, age, and consciousness impairment at presentation. J Neurol Neurosurg Psychiatry. 2006;77:611-5.

7. Liu Z, Gatt A, Mikati M, Holmes GL. Effect of temperature on kainic acidinduced seizures. Brain Res. 1993;17(631):51-8.

8. Jiang W, Duong TM, de Lanerolle NC. The neuropathology of hyperthermic seizures in the rat. Epilepsia. 1999;40:5-19.

9. Lundgren J, Smith ML, Blennow G, Siesjo BK. Hyperthermia aggravates and hypothermia ameliorates epileptic brain damage. Exp Brain Res. 1994;99:43-55.

10. Wang Y, Liu PP, Li LY, Zhang HM, Li T. Hypothermia reduces brain edema, spontaneous recurrent seizure attack, and learning memory deficits in the kainic acid treated rats. CNS Neurosci Ther. 2011;17:271-80.

11. Maeda T, Hashizume K, Tanaka T. Effect of hypothermia on kainic acidinduced limbic seizures: an electroencephalographic and 14C-deoxyglucose autoradiographic study. Brain Res. 1999;13(818):228-35.

12. Schmitt FC, Buchheim K, Meierkord H, Holtkamp M. Anticonvulsant properties of hypothermia in experimental status epilepticus. Neurobiol Dis. 2006;23:689-96

13. Kowski AB, Kanaan H, Schmitt FC, Holtkamp M. Deep hypothermia terminates status epilepticus - an experimental study. Brain Res. 2012;29(1446):119-26.

14. Corry JJ, Dhar R, Murphy T, Diringer MN. Hypothermia for refractory status epilepticus. Neurocrit Care. 2008;9:189-97.

15. Ren GP, Su YY, Tian F, Zhang YZ, Gao DQ, Liu G, et al. Early hypothermia for refractory status epilepticus. Chin Med J. 2015;20(128):1679-82.

16. Bennett AE, Hoesch RE, DeWitt LD, Afra P, Ansari SA. Therapeutic hypothermia for status epilepticus: a report, historical perspective, and review. Clin Neurol Neurosurg. 2014;126:103-9.

17. Cereda C, Berger MM, Rossetti AO. Bowel ischemia: a rare complication of thiopental treatment for status epilepticus. Neurocrit Care. 2009;10:355-8.

18. Blanco M, Campos F, Rodriguez-Yanez M, Arias S, Fernandez-Ferro J, Gomez-Sanchez JC, et al. Neuroprotection or increased brain damage mediated by temperature in stroke is time dependent. PLoS ONE. 2012; 7:e30700.

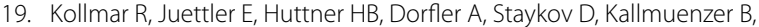
et al. Cooling in intracerebral hemorrhage $(\mathrm{CINCH})$ trial: protocol of a randomized German-Austrian clinical trial. Int J Stroke. 2012;7:168-72.

20. Kollmar R, Staykov D, Dorfler A, Schellinger PD, Schwab S, Bardutzky J. Hypothermia reduces perihemorrhagic edema after intracerebral hemorrhage. Stroke. 2010;41:1684-9.

21. Steiner T, Bosel J. Options to restrict hematoma expansion after spontaneous intracerebral hemorrhage. Stroke. 2010;41:402-9.

22. Rincon F, Lyden P, Mayer SA. Relationship between temperature, hematoma growth, and functional outcome after intracerebral hemorrhage. Neurocrit Care. 2013;18:45-53.
23. Seule MA, Muroi C, Mink S, Yonekawa Y, Keller E. Therapeutic hypothermia in patients with aneurysmal subarachnoid hemorrhage, refractory intracranial hypertension, or cerebral vasospasm. Neurosurgery. 2009;64:86-92 (discussion-3)

24. Schubert GA, Poli S, Mendelowitsch A, Schilling L, Thome C. Hypothermia reduces early hypoperfusion and metabolic alterations during the acute phase of massive subarachnoid hemorrhage: a laser-Doppler-flowmetry and microdialysis study in rats. J Neurotrauma. 2008;25:539-48.

25. Todd MM, Hindman BJ, Clarke WR, Torner JC, Weeks JB, Bayman EO, et al. Perioperative fever and outcome in surgical patients with aneurysmal subarachnoid hemorrhage. Neurosurgery. 2009;64:897-908 (discussion)

26. Andrews PJ, Sinclair HL, Rodriguez A, Harris BA, Battison CG, Rhodes JK, et al. Hypothermia for Intracranial Hypertension after Traumatic Brain Injury. N Engl J Med. 2015;17(373):2403-12.

27. Polderman $\mathrm{KH}$. Induced hypothermia and fever control for prevention and treatment of neurological injuries. Lancet. 2008;7(371):1955-69.

28. Flynn LM, Rhodes J, Andrews PJ. Therapeutic hypothermia reduces intracranial pressure and partial brain oxygen tension in patients with severe traumatic brain injury: preliminary data from the Eurotherm3235 trial. Ther Hypothermia Temp Manag. 2015;5:143-51.

29. Kochanek PM, Safar PJ. Therapeutic hypothermia for severe traumatic brain injury. JAMA. 2003;11(289):3007-9.

30. Sandestig A, Romner B, Grande PO. Therapeutic hypothermia in children and adults with severe traumatic brain injury. Ther Hypothermia Temp Manag. 2014;1(4):10-20.

31. Rossetti AO. What is the value of hypothermia in acute neurologic diseases and status epilepticus? Epilepsia. 2011;52(Suppl 8):64-6.

32. Rossetti AO, Lowenstein DH. Management of refractory status epilepticus in adults: still more questions than answers. Lancet Neurol. 2011;10:922-30.

33. Outin H, Blanc T, Vinatier I. le groupe de [Emergency and intensive care unit management of status epilepticus in adult patients and children (new-born excluded). Societe de reanimation de langue francaise experts recommendations]. Rev Neurol. 2009;165:297-305.

34. Legriel S, Bedos J, Azoulay E. Managing critically III patients with status epilepticus. New York: Springer; 2008. p. 822-36.

35. Legriel S, Resche-Rigon M, Cariou A. Dual anticonvulsant and neuroprotective effects of therapeutic hypothermia after status epilepticus. Clin Neurol Neurosurg. 2015;131:87-8.

36. Bernard SA, Gray TW, Buist MD, Jones BM, Silvester W, Gutteridge G, et al. Treatment of comatose survivors of out-of-hospital cardiac arrest with induced hypothermia. N Engl J Med. 2002;21(346):557-63.

37. Hypothermia after Cardiac Arrest Study Group. Mild therapeutic hypothermia to improve the neurologic outcome after cardiac arrest. N Engl J Med. 2002:21(346):549-56.

38. Vasile B, Rasulo F, Candiani A, Latronico N. The pathophysiology of propofol infusion syndrome: a simple name for a complex syndrome. Intensive Care Med. 2003;29:1417-25.

39. Jennett B, Teasdale G, Braakman R, Minderhoud J, Knill-Jones R. Predicting outcome in individual patients after severe head injury. Lancet. 1976;15(1):1031-4.

40. Mayer SA, Brun NC, Begtrup K, Broderick J, Davis S, Diringer MN, et al. Efficacy and safety of recombinant activated factor VII for acute intracerebral hemorrhage. N Engl J Med. 2008:15(358):2127-37.

41. van de Beek D, de Gans J, Spanjaard L, Weisfelt M, Reitsma JB, Vermeulen M. Clinical features and prognostic factors in adults with bacterial meningitis. N Engl J Med. 2004;28(351):1849-59.

42. Wilson JT, Pettigrew LE, Teasdale GM. Structured interviews for the Glasgow Outcome Scale and the extended Glasgow Outcome Scale: guidelines for their use. J Neurotrauma. 1998;15:573-85.

43. Mayer SA, Claassen J, Lokin J, Mendelsohn F, Dennis LJ, Fitzsimmons BF. Refractory status epilepticus: frequency, risk factors, and impact on outcome. Arch Neurol. 2002:59:205-10.

44. Pettigrew LE, Wilson JT, Teasdale GM. Reliability of ratings on the Glasgow Outcome Scales from in-person and telephone structured interviews. J Head Trauma Rehabil. 2003;18:252-8.

45. Mourvillier B, Tubach F, van de Beek D, Garot D, Pichon N, Georges H, et al Induced hypothermia in severe bacterial meningitis: a randomized clinical trial. JAMA. 2013;27(310):2174-83. 